\title{
Challenges and implementation aspects of switched-mode power supplies with digital control for automotive applications
}

\author{
Samuel Quenzer-Hohmuth ${ }^{1}$, Thoralf Rosahl ${ }^{2}$, Steffen Ritzmann ${ }^{2}$, and Bernhard Wicht ${ }^{1}$ \\ ${ }^{1}$ Robert Bosch Center for Power Electronics, Reutlingen University, Reutlingen, Germany \\ ${ }^{2}$ Robert Bosch GmbH, Automotive Electronics, Reutlingen, Germany \\ Correspondence to: Samuel Quenzer-Hohmuth (samuel.quenzer-hohmuth@ reutlingen-university.de) \\ Received: 18 February 2016 - Revised: 13 May 2016 - Accepted: 25 May 2016 - Published: 28 September 2016
}

\begin{abstract}
Switched-mode power supplies (SMPS) convert an input DC-voltage into a higher or lower output voltage. In automotive, analog control is mostly used in order to keep the required output voltages constant and resistant to disturbances. The design of robust analog control for SMPS faces parameter variations of integrated and external passive components. Using digital control, parameter variations can be eliminated and the required area for the integrated circuit can be reduced at the same time.

Digital control design bears challenges like the prevention of limit cycle oscillations and controller-wind-up. This paper reviews how to prevent these effects. Digital control loops introduce new sources for dead times in the control loop, for example the latency of the analog-to-digitalconverter (ADC). Dead times have negative influence on the stability of the control loop, because they lead to phase delays. Consequently, low latency is one of the key requirements for analog-to-digital-converters in digitally controlled SMPS.

Exploiting the example of a $500 \mathrm{kHz}$-buck converter with a crossover frequency of $70 \mathrm{kHz}$, this paper shows that the $5 \mu$ s-latency of a $\Delta \Sigma$-analog-to-digital-converter leads to a reduction in phase margin of $126^{\circ}$. The latency is less critical for boost converters because of their inherent lower crossover frequencies.

Finally, the paper shows a comparison between analog and digital control of SMPS with regard to chip area and test costs.
\end{abstract}

\section{Introduction}

The increasing need for cost pressure for automotive manufacturers and suppliers leads to steadily rising requirements for various electronics components in cars. The components should get smaller, lighter and cheaper while maintaining equal performance. This affects also switched-mode power supplies, which are installed for the generation of voltage supplies with different voltage levels in cars. In order to offer a small-sized realization of SMPS there are numerous approaches. There are attempts to tune the switching frequency in order to reduce the size of the discrete components such as the inductor (Wittmann and Wicht, 2013). There are also attempts to improve the dynamic behavior of the closed loop system using digital control. This is one of the reasons why digital control of SMPS experiences growing demand.

Digital control offers several advantages compared to ana$\log$ control. Digital control is not restricted to pure linear control like analog control is. He and Xu (2007) have shown that nonlinear control has dynamic advantages compared to linear control and enables rapid compensation of load transients.

Parameter variations of compensation devices, which are necessary to adjust the dynamic behavior of an analog controller, and parameter variations of the LC-filter lead to difficulties designing the analog control. Varying values of the compensation devices result in uncontrolled variations in frequency characteristics of the analog controller. With a digital control there are no compensation devices and thus no variations in frequency behavior. Furthermore, in an integrated solution chip size and thereby costs decrease.

The still present variations of the LC-filter can be compensated in digital control by establishing a concept for stabiliz- 
ing the crossover frequency and phase margin of the closedloop system, as presented by Morroni et al. (2009).

This paper presents a digitally controlled boost converter and shows why digital control for a buck converter with a $\Delta \Sigma$-analog-to-digital-converter with $5 \mu$ s-latency is not achievable. Besides, it shows how to prevent limit cycle oscillations (LCOs) and controller wind-up. A comparison of chip size and test costs of the digitally controlled boost converter with an analog controlled boost converter concludes the paper.

\section{Challenges and implementation aspects}

In order to digitally control a SMPS, additional components are required, namely an analog-to-digital-converter and a digital-to-analog-converter (DAC). In return, the realization of the controller is easier and needs less hardware (no compensation devices) than an analog controller. With these additional components the control loop is built as shown in Fig. 1. Signal $X$ corresponds to the output voltage of a SMPS which is typically scaled down to a lower voltage with a resistor feedback divider and then converted into a digital value $\hat{X}$. Signal $\hat{Y}$ is the digital controller output value. $Y$ correlates to the PWM signal which is necessary to control the switch of the SMPS.

\subsection{Phase delays}

In digital control loops there are sources for dead times, for example the latency of the analog-to-digital-converter and of the digital-to-analog-converter or the calculation time of the digital controller. The sampling with finite sample time can be modeled with a correlating dead time.

Dead times degrade the stability of the control loop because they introduce unwanted phase shifts. As a result, dead times reduce the phase margin, which causes a less damped or even unstable control loop. The relation of dead time $T_{\mathrm{d}}$ and phase shift $\Delta \varphi$ shows Eq. (1):

$\Delta \varphi=-\omega \cdot T_{\mathrm{d}}$

\subsubsection{ADC and DAC caused dead times}

Analog-to-digital-converters need time for the conversion of the analogous input voltage to a digital output value. The same applies for the digital-to-analog-converter. Depending on the converter architecture greater or smaller dead times result. This latency appears as dead time for the control loop. Another source of a dead time is the calculation time of the controller output value.

\subsubsection{Sampling caused delay}

In a digital control circuit the output signal is sampled and processed from the controller. Afterwards it is hold till the

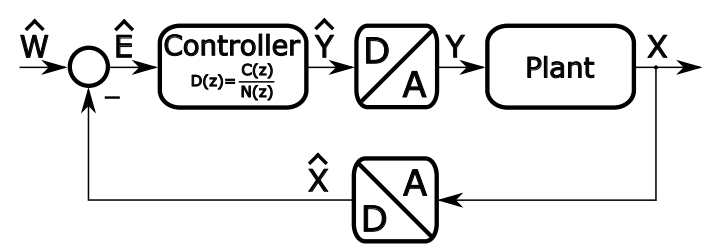

Figure 1. Digital control circuit

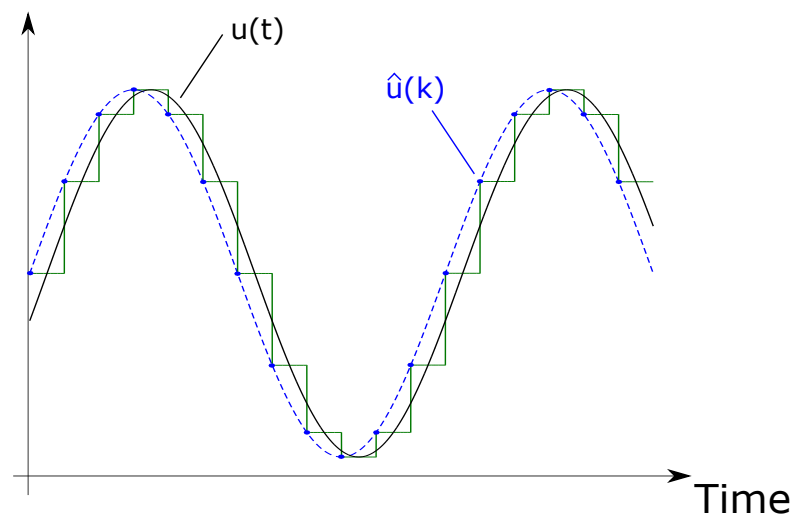

Figure 2. Sampling caused delay

next sample is taken and processed. This behavior is modeled with a zero-order-hold. Since the zero-order-hold is not a linear time-invariant system, there is no possibility to determine a transfer function. But with the help of a sinusoidal stimulus and the help of the resulting signal response it is possible to find a proper model for the zero-order-hold (Lunze, 2010).

If the input signal of the zero-order-hold is the sinusoidal sequence $\hat{u}(k)=\sin (k T)$, it will generate a continuous step function as shown in Fig. 2.

The input of the controlled system is the step function, which contains a wide spectrum of frequencies. Most controlled systems have low-pass characteristics, thus the high frequencies of the step function will not be transmitted and can therefore be disregarded for modeling. The small frequencies (especially the fundamental frequency) have to be considered. The fundamental frequency after Fourier analysis, in case the sampling theorem is satisfied, can be described as Eq. (2) shows.

$u(t)=\frac{2 \cdot \sin (0.5 \cdot T)}{T} \cdot \sin \left(t-T_{\mathrm{d}}\right)$

This shows that the zero-order-hold has a gain, which is nearby 1 and hence, not relevant. On the other hand there is a significant delay. The resulting delay $T_{\mathrm{d}}$ as a function of the sampling rate $T$ is approximately:

$T_{\mathrm{d}}=\frac{T}{2}$ 


\subsection{Limit cycle oscillations}

The quantization introduced through the ADC and the DAC can lead to limit cycle oscillations. Limit cycle oscillations are steady-state oscillations of the output voltage at lower frequencies as the switching frequency of the converter (Peterchev and Sanders, 2001).

\subsubsection{Resolution caused LCOs}

Due to the quantization of the output voltage through the analog-to-digital-converter there is an analogous voltage range with the width of one LSB (hereinafter called "bin") for each digital value. Thus there is an analogous voltage bin for the digital set point (0-bit-error-bin). If the output voltage is located in this bin, the control error will be zero. Premise for permanent elimination of the control error is that there is a digital-to-analog-converter value (DAC-value) which forces the output voltage to stay within the 0-bit-error-bin. To fulfill this premise the resolution of the digital-to-analog-converter referred to the output voltage has to be higher than the resolution of the analog-to-digital-converter referred to the output voltage.

Resolution (DAC) > Resolution (ADC)

If this condition is not met, there will not be a DAC-value for every output voltage bin. Figure 3 shows what will happen if the above mentioned condition is not taken into account. There is no DAC-value which leads to a voltage within the 0 -bit-error-bin. That is why the output voltage $V_{\text {out }}$ does not stay permanently within the 0-bit-error-bin and a limit cycle oscillation arises.

\subsubsection{Missing integral term caused LCOs}

If the controller has no integral term, limit cycle oscillations can occur regardless of the condition given in Eq. (4). This issue is shown in Fig. 4. As long as there is a control error the controller tries to drive the output voltage $V_{\text {out }}$ towards the 0 bit-error-bin. As soon as the output voltage reaches the 0-biterror-bin the control error gets zero. Without integral term in the controller the DAC-value changes immediately and as a consequence the output voltage leaves the 0-bit-error-bin again.

To avoid this kind of limit cycle oscillations the controller requires to have an integral term.

\subsubsection{No limit cycle oscillations}

Beside the cases shown in this paper, Peterchev and Sanders (2001) as well as Peng et al. (2004) show further special cases leading to limit cycle oscillations.

If all conditions for no limit cycle oscillations are met, the output voltage will stay within the 0-bit-error-bin in steadystate. If the controller has an integral term, the digital value

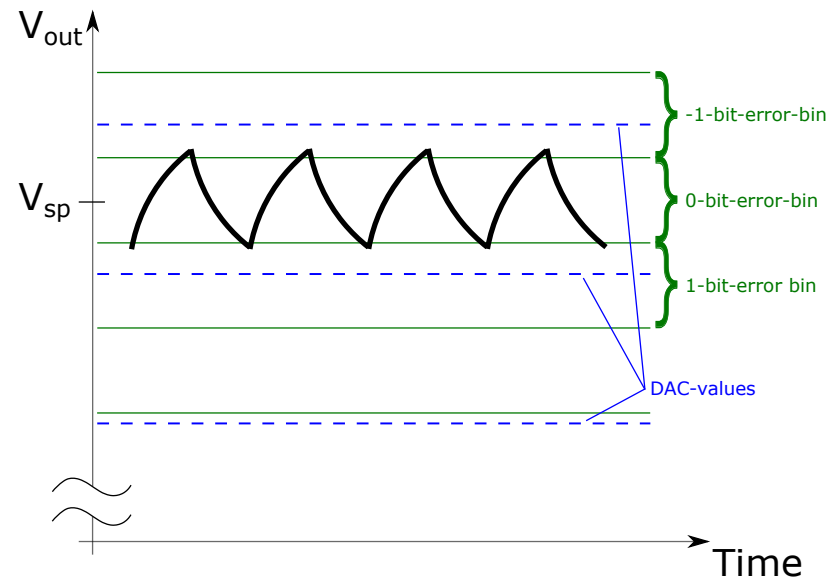

Figure 3. Resolution caused limit cycle oscillations

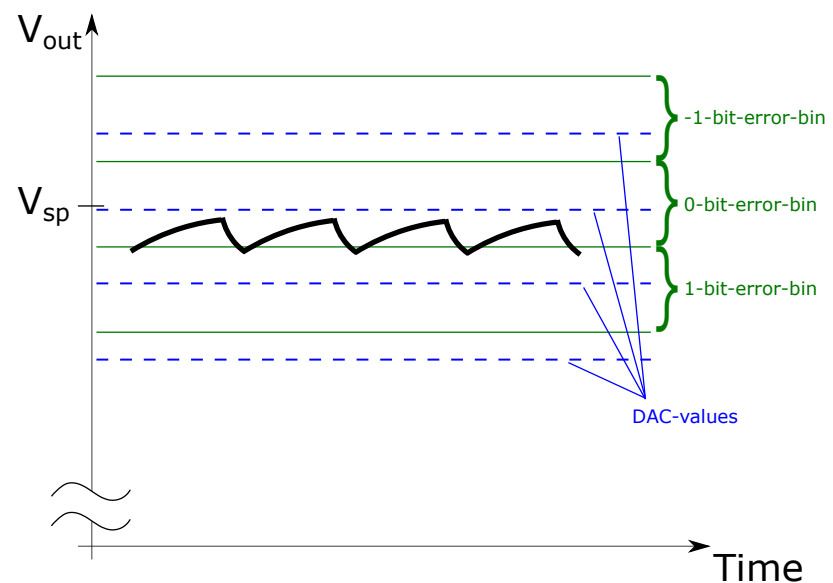

Figure 4. Missing integral term caused limit cycle oscillations

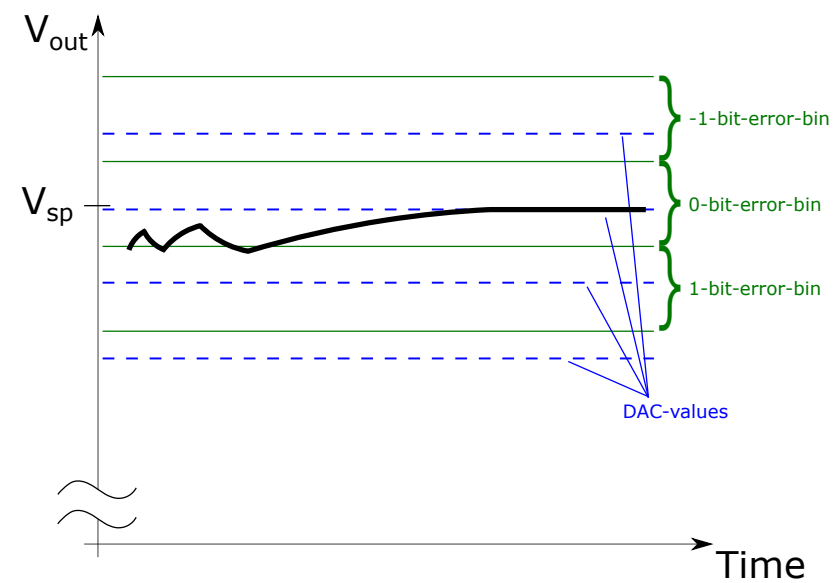

Figure 5. No limit cycle oscillations

will be approximated to the right value step by step. Figure 5 shows this approximation process of the output voltage $V_{\text {out }}$. 


\subsection{Controller wind-up}

In a digital control loop controller wind-up is possible if the controller has an integral term, which is necessary for avoiding limit cycle oscillations. This unwanted effect can be eliminated using a dedicated anti-wind-up controller structure.

\subsubsection{Controller wind-up mechanism}

In a digital control loop (see Fig. 1) the digital controller output signal is converted by a digital-to-analog-converter into an analogous signal. This DAC has an input range, hence the DAC-value cannot get as high nor as low as it would be required. The value calculated from the controller can thus be higher than the DAC-value appearing at the plant. Consequently, in case of large control deviation the output voltage does not move back to the set point as fast as the controller scheduled it.

The controller integrates the control deviation and the calculated value now gets even higher than before. But the digital-to-analog-converter still does not allow the DACvalue seen from the plant to get any higher.

The controller further integrates until the control deviation changes in sign. This happens because the controller has counteracted the control deviation, the DAC has only limited the power to do so. The large controller output value needs to be integrated into the opposite direction to get back into the input range of the DAC. This delaying effect leads to long lasting and huge control deviations.

\subsubsection{Anti-wind-up controller structure}

Controller-wind-up can be counteracted with an anti-windup controller structure. The anti-wind-up controller structure, as shown in Fig. 6, is modified marginally compared to a standard controller.

The original controller $D(z)$ is divided in numerator and denominator $D(z)=\frac{\hat{Y}(z)}{\hat{E}(z)}=\frac{C(z)}{N(z)}$. With this partitioning the anti-wind-up controller structure can be built as shown in Fig. 6 with only one more block compared to a standard architecture (Fig. 1). This block is a limitation block with the limits matching to the limits of the digital-to-analogconverter (or any other limiting element).

It can be shown that $\hat{V}(z)$ corresponds to the original controller output signal in the case that $\hat{V}(z)$ is within the limits of the limitation block. Then $\hat{V}(z)=\hat{Y}(z)$ is valid.

$\hat{V}(z)=\hat{V}(z) \cdot(1-N(z))+C(z) \cdot \hat{E}(z)$

By manipulating the equation, it can be shown that this modified anti-wind-up controller structure matches to the original controller.

$D(z)=\frac{\hat{Y}(z)}{\hat{E}(z)}=\frac{C(z)}{N(z)}$

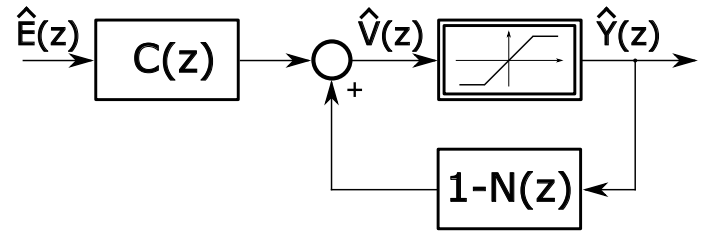

Figure 6. Anti-wind-up controller structure

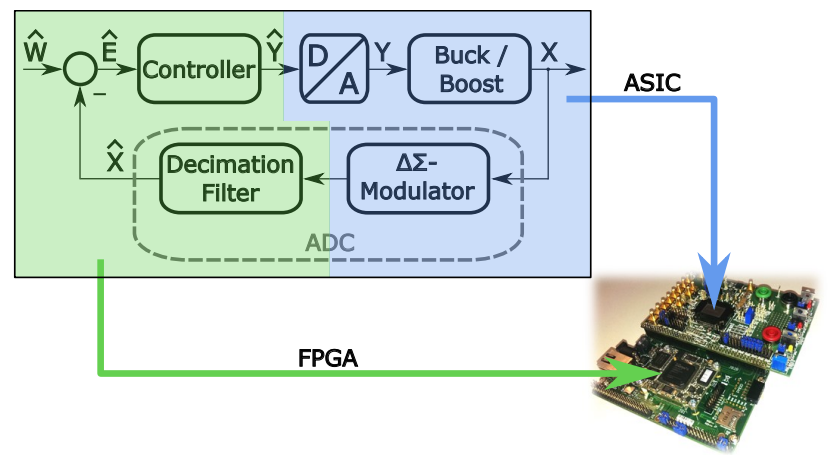

Figure 7. Measurement setup for SMPS with digital control

If $\hat{V}(z)$ crosses a limit of the limitation block (and thus the limit of the digital-to-analog-converter) this internal limitation block prohibits further growth of the controller output. Further integration is stopped although a control deviation is still existent. Once the control deviation changes in sign the controller reacts immediately (in contrast to the standard controller architecture).

Further information on controller wind-up can be found in Schulz and Graf (2013).

\section{Digitally controlled switched-mode power supplies}

The hardware for the experimental results in this paper consists of two main components, an application specific integrated circuit (ASIC) produced in a $110 \mathrm{~nm}$-technology and a Xilinx Spartan ${ }^{\circledR}-6$ field programmable gate array (FPGA), as shown in Fig. 7. The ASIC contains the digital-to-analogconverter, the plant, thus either a buck or a boost converter, and a $\Delta \Sigma$-modulator. All digital parts are implemented on the FPGA: Decimation filter of the analog-todigital-converter and digital controller. Further information on the parameters of the analog-to-digital-converter and the digital-to-analog-converter can be found in Table 1. 
Table 1. Parameters of ADC and DAC

\begin{tabular}{lll}
\hline Parameter & ADC & DAC \\
\hline Architecture: & $\Delta \Sigma$ & R-2R \\
Resolution: & $10 \mathrm{bit}$ & $10 \mathrm{bit}$ \\
& $18 \mathrm{mV} \mathrm{LSB}^{-1}$ & $12 \mathrm{mV} \mathrm{LSB}^{-1}$ \\
Latency: & $5 \mu \mathrm{s}$ & $\mathrm{n} / \mathrm{a}$ \\
Sampling rate: & $400 \mathrm{kSps}$ & $400 \mathrm{kSps}$ \\
\hline
\end{tabular}

Table 2. Parameters of buck and boost converter

\begin{tabular}{lll}
\hline Parameter & Buck & Boost \\
\hline Input voltage: & $14 \mathrm{~V}$ & $3.5 \mathrm{~V}$ \\
Output voltage: & $6 \mathrm{~V}$ & $6.3 \mathrm{~V}$ \\
Switching frequency: & $500 \mathrm{kHz}$ & $500 \mathrm{kHz}$ \\
Crossover frequency: & $70 \mathrm{kHz}$ & $2 \mathrm{kHz}$ \\
\hline
\end{tabular}

Table 2 presents information about the boost and buck converter.

\subsection{Buck converter}

As the latency of the analog-to-digital-converter is $T_{\mathrm{d}}=5 \mu \mathrm{s}$ the phase delay for a crossover frequency of $f_{\mathrm{c}}=70 \mathrm{kHz}$ can be calculated with Eq. (1).

$\Delta \varphi=-\omega \cdot T_{\mathrm{d}}=-2 \cdot 180^{\circ} \cdot 70 \mathrm{kHz} \cdot 5 \mu \mathrm{s}=-126^{\circ}$

In addition there is the inherent delay through sampling. With Eq. (2) the effective dead time results in $T_{\mathrm{d}}=T / 2=$ $1.25 \mu \mathrm{s}$. Accordingly, the phase delay gets $\Delta \varphi=-\omega \cdot T_{\mathrm{d}}=$ $-2 \cdot 180^{\circ} \cdot 70 \mathrm{kHz} \cdot 1.25 \mu \mathrm{s}=-31.5^{\circ}$. Adding the two phase delays the total phase delay reaches $\Delta \varphi_{\mathrm{tot}}=-157.5^{\circ}$. This total phase delay is very high and therefore digital control for a buck converter with $70 \mathrm{kHz}$ crossover frequency and with the available $5 \mu$ s-latency analog-to-digital-converter is not possible. This buck converter with digital control could either be realized with a low-latency ADC or an ADC dedicated for digital control of SMPS, as proposed by Lukic et al. (2007).

\subsection{Boost converter}

In comparison to the buck converter, the boost converter has an inherent lower crossover frequency because of its right-half-plane zero (Basso, 2008). Thus the latency of the analog-to-digital-converter has less influence on the phase delay $\Delta \varphi=-\omega \cdot T_{\mathrm{d}}=-2 \cdot 180^{\circ} \cdot 2 \mathrm{kHz} \cdot 5 \mu \mathrm{s}=-3.6^{\circ}$. Accordingly, the sampling caused phase delay is less, too: $\Delta \varphi=$ $-\omega \cdot T_{\mathrm{d}}=-2 \cdot 180^{\circ} \cdot 2 \mathrm{kHz} \cdot 1.25 \mu \mathrm{s}=-0.9^{\circ}$. The total phase delay is $\Delta \varphi_{\mathrm{tot}}=-4.5^{\circ}$ and, consequently, digital control for a boost converter is possible without major impact on stability.

Figure 8 shows measurement results for the presented boost converter in case of a load transient. The output voltage

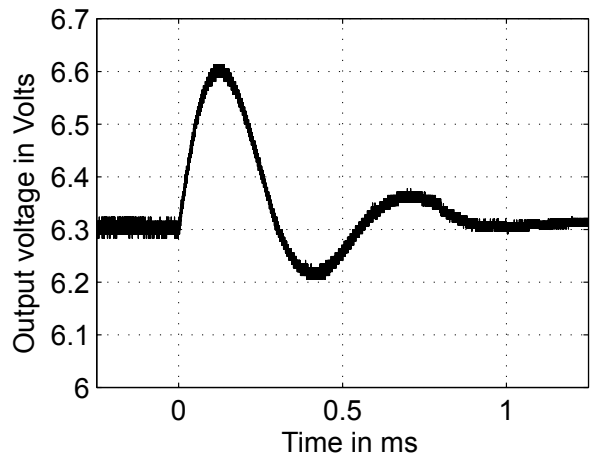

Figure 8. Measured load transient of the presented boost converter $\left(I_{\text {load }}=160 \mathrm{~mA} \rightarrow I_{\text {load }}=60 \mathrm{~mA}\right)$

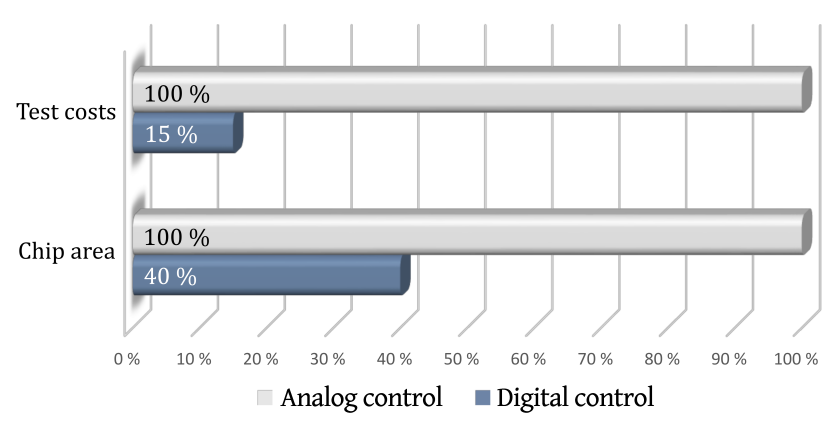

Figure 9. Test costs and chip area of digital and analog control in comparison

recovers safely from the load transient, thus, the latency has no impact on the stability of the boost converter.

\section{Comparison between analog and digital control}

This section shows a comparison of chip area and test costs necessary for the integration of the control for a boost converter. The comparison is elaborated on the one hand for an analog control used in a conventional automotive chip and, on the other hand, for a digital control with comparable characteristics. The comparison does not consider the total area of the converter, it considers only the diverging parts in analog and digital control. Thus, for digital control only ADC, digital controller and DAC are considered. For the analog control only the error amplifier including compensation components (two capacitors and one ohmic resistor) are considered. These components are necessary in analog control to realize a controller with the same frequency characteristic as the presented digital controller.

\subsection{Test costs}

The test costs are derived from the test time. Figure 9 illustrates test costs for the analog control, normalized to $100 \%$. The test costs for the digital control are given relative to the 
test costs for the analog control. The tests for the analog control involve especially error amplifier testing, for example measurement of the transfer characteristic and measurement of the operating range. For the digital control, typical digital tests like stuck-at-fault and Iddq-measurements are performed. The results show that test costs for the digital control are $85 \%$ lower, compared to analog control.

\subsection{Chip area}

The considered digital control offers more flexibility than the analog control. The digital controller coefficients are implemented to be flexible. Area effort is much higher for variable coefficients than for fixed coefficients. In return, variable coefficients allow to change the dynamic behavior of the control loop in operation.

Figure 9 shows that the implementation area of the digital control of a boost converter is significantly smaller than the layout area of the analog control of a fully-integrated boost converter (incl. passives of the control loop). Furthermore, the digital control offers more dynamic flexibility in operation.

\section{Conclusions}

This paper presents that limit cycle oscillations can be prevented by securing that the resolution of the DAC is higher than the resolution of the ADC and by implementing a controller with integral term. Furthermore, controller-windup can be prevented by the implementation of a dedicated anti-wind-up controller structure with a limiter block. Digital control circuits contain sources for latency, such as the analog-to-digital-converter and the digital-to-analogconverter. Latencies impact the stability of the control loop. The example of a $500 \mathrm{kHz}$-buck converter with a crossover frequency of $70 \mathrm{kHz}$ shows that digital control cannot be realized with a $5 \mu$ s-latency $\Delta \Sigma$-analog-to-digital-converter. Consequently, a low latency is one of the key requirements for analog-to-digital-converters. A comparison of analog and digital control indicates that chip area (60\%-reduction) and test costs (85\%-reduction) for switched-mode power supplies can be reduced by digital control. At the same time flexibility in operation can be achieved with variable controller coefficients. Thus, adaptive, nonlinear, and dynamic-optimized control can be realized. Switched-mode power supplies with digital control show high potential for value-added power management systems in automotive ICs.

Edited by: D. Killat

Reviewed by: two anonymous referees

\section{References}

Basso, C. P.: Switch-Mode Power Supplies: SPICE Simulations and Practical Designs, The McGraw-Hill Companies, New York, USA, 2008.

$\mathrm{He}$, M. and $\mathrm{Xu}$, J.: Nonlinear PID in Digital Controlled Buck Converters, in Applied Power Electronics Conference, APEC 2007 - Twenty Second Annual IEEE, 1461-1465, doi:10.1109/APEX.2007.357709, 2007.

Lukic, Z., Rahman, N., and Prodic, A.: Multibit Sigma-Delta PWM Digital Controller IC for DC-DC Converters Operating at Switching Frequencies Beyond $10 \mathrm{MHz}$, IEEE T. Power Electr., 22, 1693-1707, doi:10.1109/TPEL.2007.904199, 2007.

Lunze, J.: Regelungstechnik 2, Springer Verlag, Berlin Heidelberg, Germany, 2010.

Morroni, J., Zane, R., and Maksimović, D.: Design and Implementation of an Adaptive Tuning System Based on Desired Phase Margin for Digitally Controlled DC-DC Converters, IEEE T. Power Electr., 24, 559-564, doi:10.1109/TPEL.2008.2007641, 2009.

Peng, H., Maksimović, D., Prodić, A., and Alarcón, E.: Modeling of Quantization Effects in Digitally Controlled DC-DC Converters, IEEE 35th Annual Power Electronics Specialists Conference, 2004 (PESC), 6, 4312-4318, doi:10.1109/PESC.2004.1354763, 2004.

Peterchev, A. V. and Sanders, S. R.: Quantization Resolution and Limit Cycling in Digitally Controlled PWM Converters, IEEE 32nd Annual Power Electronics Specialists Conference, 2001 (PESC), 2, 465-471, doi:10.1109/PESC.2001.954158, 2001.

Schulz, G. and Graf, K.: Mehrgroessenregelung, Digitale Regelungstechnik, Fuzzy-Regelung, Oldenbourg, Munich, Germany, 2013.

Wittmann, J. and Wicht, B.: MHz-Converter Design for High Conversion Ratio, IEEE 25th International Symposium on Power Semiconductor Devices and ICs (ISPSD), 127-130, doi:10.1109/ISPSD.2013.6694445, 2013. 